\title{
Plant Growth Parameters and Soil Potassium Pool as Influenced by Potassium Fertilization in Kharif Season Rice
}

\author{
Sai Parasar Das*, Goutam Kumar Ghosh, Suchhanda Mondal, \\ Pabitra Kumar Biswas and Manik Chandra Kundu
}

Department of Soil Science and Agricultural Chemistry, Institute of Agriculture, Visva-Bharati, Sriniketan, West Bengal (731 236), India

*Corresponding author:

\begin{abstract}
A B S T R A C T
Keywords

Soil potassium pool,

Plant parameters,

Potassium

fertilization

Article Info

Accepted:

12 February 2019

Available Online:

10 March 2019

\section{Introduction}

Attaining Food Security had been a major challenge for the nation since independence. With ever increasing population it is very crucial for our country to increase food grain production from the current level. United Nations Sustainable Development Goal no. 2 also talks about increasing agro productivity two fold by the end of 2030. The current stagnation in food grain production necessitates special initiatives to meet the increasing demands of food grains. Expansion
\end{abstract}

The field experiment was conducted during kharif season of 2016-17 and 2017-18 to find the influence of potassium fertilization on different growth and yield parameters of rice along with soil potassium pool. The experiment was laid out in Randomized Block Design with treatments comprised of different doses of potassium viz. 15, 30, 45, 60, 90, 120 $\mathrm{kg} / \mathrm{ha}$ and a control plot without any fertilizer and it was replicated thrice. The experiment revealed that the plant growth parameters viz. plant height, number of active tillers per plant, test weight and yield were significantly increased with application of potassium fertilizer. With application of potassium fertilizer, plant parameters such as plant height, no of active tillers, test weight and yield of rice increased significantly. Pooled data of both the years suggested that, water soluble, exchangeable, available and $\mathrm{HNO}_{3}$ soluble potassium increased at all three stages, as fertilizer dose increased. The exchangeable potassium in control plot showed a decreasing trend with time. of the area has ceased to be a major source of increased output. Most of the targeted increase in production now must result from greater yield per hectare. Appropriate nutrient management in general and potassium management in particular, will play a major role in overcoming stagnation in crop production. The picture of crop responses to potassium $(\mathrm{K})$ in India has been changing with time. Although Indian soils were sufficient in terms of potassium status but intensive cropping without its application has rendered the soils deficient in potassium. There is a 
growing evidence of increasing deficiency of $\mathrm{K}$ as a result of inadequate use of the nutrient as compared to nitrogen $(\mathrm{N})$ and phosphorus (P). International potash institute found $\mathrm{K}$ balance negative in many soils even under the optimum rates of fertilization.

In view of the above considerations, the experiment was conducted to characterize the soil with respect to potassium status and determine different forms of potassium in soil and its dynamics.

\section{Materials and Methods}

The field experiment was conducted in the kharif season of 2016-17 and repeated the same in the year 2017-18 at the Agricultural Farm of PalliSiksha Bhavana (Institute of Agriculture), Visva- Bharati, Sriniketan which situated at $23^{\circ} 39^{\prime} \mathrm{N}$ latitude and $87^{\circ} 42^{\prime} \mathrm{E}$ longitude with an average altitude of $58.9 \mathrm{~m}$ above the mean sea level under sub humid semi-arid region of West Bengal.

The experiment was conducted in a randomized block design having three replications in both the years. Seven different treatments were randomly allotted to the plots following random number table (Gomez and Gomez, 1976)

The experiment was laid out in Randomized Block Design with three replications in $6 \mathrm{~m} \mathrm{x}$ $5 \mathrm{~m}$ plots and spacing of $20 \mathrm{~cm} . \times 10 \mathrm{~cm}$. The treatment comprised of different doses of potassium viz. 15,30, 45, 60, 90, $120 \mathrm{~kg} / \mathrm{ha}$ along with a control plot without having any fertilizer applied.

Height of plants and number of tillers per plant was measured by taking measurement of 5 plants from each plot was at 45, 70 and 90 DAT. A sample of 1000 seeds was drawn randomly from each plot. The sample was weighed and analyzed statistically. After harvesting was done from individual plot, the grain was weighed with balance and yield per hectare was calculated.

Water soluble potassium was determined by following procedure of Black et al., (1965) by taking soil and water in 1:2 ratio, shaking on the mechanical shaker for two hours and allowing it to reach equilibrium for additional sixteen hours. Available potassium of soil samples was determined in soil: neutral normal ammonium acetate extract (1:5) of the soil using flame photometer (Jackson 1973). Exchangeable $\mathrm{K}$ was the difference between available and water soluble potassium. Nitric acid soluble potassium was determined by following procedure of Wood and Deturk (1941).

\section{Results and Discussion}

\section{Plant parameters in rice}

\section{Plant height}

The observations on plant height each year, recorded at 45, 70, and 90 days after transplanting (DAT) were analyzed statistically and presented in the table no 1.The plant height of kharif rice was highly influenced by the application of potassic fertilizer in every stage i.e. 45, 70, and 90days after transplanting (DAT).

At 45 DAT the height increased from control $\left(\mathrm{T}_{1}\right)$ to $\mathrm{T}_{5}$ having potassium application rate $60 \mathrm{~kg} / \mathrm{ha}$ then declined. At 70 DAT and 90 DAT height increased with increase in fertilizer dose. Highest value was recorded in $\mathrm{T}_{7}$ treatment which was significantly higher than other treatments except $\mathrm{T}_{4}, \mathrm{~T}_{5}, \mathrm{~T}_{6}$, with which it was at par. Similar effect of potassium fertilization was reported by Bahmaniar et al., (2007), Biswas et al., (2001) and Mukherjee and Sen (2005). 


\section{No. of active tillers/hill}

A perusal of pooled data shows that no. of active tillers per hill was highly influenced by potassium fertilization. Highest no. of active tillers were recorded in treatment $T_{7}$ followed by $T_{5}$ and $T_{6}$ and was significantly higher than rest of the treatments (table no 2). Similar results were corroborated by Thakur et al., (1993), Meena et al., (2003) and Tabar et al., (2012).

\section{Test weight}

The test weight data from different years were pooled together where it can be noticed that test weight increased with increasing the dose of potassium fertilizer. Highest test weight was recorded in treatment $T_{7}$ which was at par with the all treatments except control with which it was significantly higher. The pooled data revealed that the range of test weight was from 11.94 to $15.47 \mathrm{~g}$.(table no 2).

\section{Grain yield}

Grain yield was also influenced by fertilization as revealed from table no 2 . It ranged from 2.04 to $3.24 \mathrm{t} \mathrm{ha}^{-1}$ and 1.94 to $3.40 \mathrm{t} \mathrm{ha}^{-1}$ for years 2016-17 and 2017-18 respectively. The pooled data revealed that highest yield was recorded from treatment $T_{5}$ which was significantly higher than treatment $\mathrm{T}_{1}$ and $\mathrm{T}_{2}$. The second highest yield was recorded in treatment $T_{6}$ and $T_{7}$ followed by treatment $\mathrm{T}_{4}$. Krishnappa et al., (2006) revealed that potassium application had positive impact on yield of rice. Similar observations were reported by Mathad et al., (2002) and Biswas et al., (2001).

\section{Soil potassium pool}

\section{Water soluble potassium}

Soil samples from surface soils were analyzed for water soluble potassium at different intervals of kharif season rice for successive years 2016-17 and 2017-18 and pooled together in table no 3 . At 45 DAT highest water soluble potassium was present in treatment of highest dose of fertilizer application, $\mathrm{T}_{7}$ and was significantly higher than all other treatments. Similar trends were seen during various stages of crop 70 DAT and $90 \mathrm{DAT}$. The range of water soluble potassium was in the range of 10.04 to 23.20 ppm, 9.64 to $22.97 \mathrm{ppm}$ and 8.86 to 22.36 ppm for 45 DAT, 70 DAT and 90 DAT respectively. The control plot showed a different trend than other treatments where water soluble potassium decreased with passing of time, while for the rest of the treatment this trend was absent due to fertilization. Similar results were revealed from experiments by Padole and Mahajan (2003), Ranganathan and Sathyanarayana (1980) and Pharande and Sonar (1996). The quantity of water soluble $\mathrm{K}$ was found lower than other potassium fractions which could be due to the fact that $\mathrm{K}$ in solution is present in lower quantities in comparison to other potassium reserves (Bansal et al., 2002).

\section{Exchangeable potassium}

Highest exchangeable potassium was found in $\mathrm{T}_{7}$ treatment at all three stages $45,70,90 \mathrm{DAT}$ i.e. $79.95,80.96,76.71 \mathrm{ppm}$ respectively and the values were significantly higher than all other treatments except treatment $\mathrm{T}_{5}$ and $\mathrm{T}_{6}$ with which it was at par. Lowest value was recorded in control plot having no potassium application (table no 4). Pooled data suggests that, at all three stages exchangeable potassium increased as fertilizer dose increased. The exchangeable potassium in control plot showed decreasing trend with time which was due to the depletion of potassium pool with plant uptake and leaching. Similar trend was observed from the pooled data of all the treatments except treatment $\mathrm{T}_{7}$ where no such trend was discernible. 
Table.1 Effect of potassium fertilizer application on plant height (in $\mathrm{cm}$ ) in Kharif Rice

\begin{tabular}{|l|l|l|l|l|l|l|l|l|l|}
\hline & \multicolumn{3}{|c|}{ 45 DAT } & \multicolumn{3}{c|}{ 70 DAT } & \multicolumn{3}{c|}{ 90 DAT } \\
\hline Treatments & $2016-17$ & $2017-18$ & Pooled & $2016-17$ & $2017-18$ & Pooled & $2016-17$ & $2017-18$ & Pooled \\
\hline $\mathbf{T}_{\mathbf{1}}\left(\mathbf{N}_{\mathbf{6 0}} \mathbf{P}_{\mathbf{3 0}} \mathbf{K}_{\mathbf{0}}\right)$ & 36.82 & 35.67 & 36.25 & 72.32 & 71.56 & 71.94 & 99.76 & 101.27 & 100.52 \\
\hline $\mathbf{T}_{\mathbf{2}}\left(\mathbf{N}_{\mathbf{6 0}} \mathbf{P}_{\mathbf{3 0}} \mathbf{K}_{\mathbf{1 5}}\right)$ & 40.73 & 39.28 & 40.00 & 76.27 & 74.73 & 75.50 & 108.81 & 108.21 & 108.51 \\
\hline $\mathbf{T}_{\mathbf{3}}\left(\mathbf{N}_{\mathbf{6 0}} \mathbf{P}_{\mathbf{3 0}} \mathbf{K}_{\mathbf{3 0}}\right)$ & 41.21 & 40.21 & 40.71 & 80.94 & 79.86 & 80.40 & 109.53 & 111.27 & 110.40 \\
\hline $\mathbf{T}_{\mathbf{4}}\left(\mathbf{N}_{\mathbf{6 0}} \mathbf{P}_{\mathbf{3 0}} \mathbf{K}_{\mathbf{4 5}}\right)$ & 42.31 & 41.75 & 42.03 & 79.46 & 77.42 & 78.44 & 111.79 & 111.00 & 111.40 \\
\hline $\mathbf{T}_{\mathbf{5}}\left(\mathbf{N}_{\mathbf{6 0}} \mathbf{P}_{\mathbf{3 0}} \mathbf{K}_{\mathbf{6 0}}\right)$ & 45.59 & 44.19 & 44.89 & 79.01 & 82.07 & 80.54 & 112.85 & 113.71 & 113.28 \\
\hline $\mathbf{T}_{\mathbf{6}}\left(\mathbf{N}_{\mathbf{6 0}} \mathbf{P}_{\mathbf{3 0}} \mathbf{K}_{\mathbf{9 0}}\right)$ & 45.72 & 43.75 & 44.73 & 83.37 & 79.65 & 81.51 & 113.60 & 113.10 & 113.35 \\
\hline $\mathbf{T}_{\mathbf{7}}\left(\mathbf{N}_{\mathbf{6 0}} \mathbf{P}_{\mathbf{3 0}} \mathbf{K}_{\mathbf{1 2 0}}\right)$ & 43.23 & 42.05 & 42.64 & 85.55 & 79.30 & 82.42 & 112.52 & 117.19 & 114.85 \\
$\mathbf{S} \mathbf{E m}(\mathbf{\pm}$ & 1.20 & 1.34 & 0.90 & 2.10 & 2.23 & 1.55 & 2.92 & 3.03 & 2.17 \\
\hline $\mathbf{C D}$ at 5\% & 3.71 & 4.12 & 2.63 & 6.48 & 6.88 & 4.52 & 9.01 & 9.33 & 6.32 \\
\hline
\end{tabular}

Table.2 Effect of potassium fertilizer application on number of active tillers/hill, test weight and grain yield in kharif rice

\begin{tabular}{|l|l|l|l|l|l|l|l|l|l|}
\hline & \multicolumn{3}{|c|}{ No. of Active Tillers/hill } & \multicolumn{3}{c|}{ Test weight (in g) } & \multicolumn{3}{c|}{ Grain Yield (in t/ha) } \\
\hline Treatments & $2016-17$ & $2017-18$ & Pooled & $2016-17$ & $2017-18$ & Pooled & $2016-17$ & $2017-18$ & Pooled \\
\hline $\mathbf{T}_{\mathbf{1}}\left(\mathbf{N}_{\mathbf{6 0}} \mathbf{P}_{\mathbf{3 0}} \mathbf{K}_{\mathbf{0}}\right)$ & 8.74 & 9.97 & 9.35 & 13.69 & 10.18 & 11.94 & 2.04 & 1.94 & 1.99 \\
\hline $\mathbf{T}_{\mathbf{2}}\left(\mathbf{N}_{\mathbf{6 0}} \mathbf{P}_{\mathbf{3 0}} \mathbf{K}_{\mathbf{1 5}}\right)$ & 10.93 & 11.70 & 11.32 & 15.23 & 14.09 & 14.66 & 2.59 & 2.31 & 2.45 \\
\hline $\mathbf{T}_{\mathbf{3}}\left(\mathbf{N}_{\mathbf{6 0}} \mathbf{P}_{\mathbf{3 0}} \mathbf{K}_{\mathbf{3 0}}\right)$ & 12.20 & 13.80 & 13.00 & 15.21 & 14.46 & 14.84 & 2.98 & 3.01 & 2.99 \\
\hline $\mathbf{T}_{\mathbf{4}}\left(\mathbf{N}_{\mathbf{6 0}} \mathbf{P}_{\mathbf{3 0}} \mathbf{K}_{\mathbf{4 5}}\right)$ & 12.85 & 13.47 & 13.16 & 15.12 & 14.22 & 14.67 & 3.18 & 3.33 & 3.26 \\
\hline $\mathbf{T}_{\mathbf{5}}\left(\mathbf{N}_{\mathbf{6 0}} \mathbf{P}_{\mathbf{3 0}} \mathbf{K}_{\mathbf{6 0}}\right)$ & 15.05 & 15.47 & 15.26 & 15.56 & 14.97 & 15.27 & 3.24 & 3.39 & 3.32 \\
\hline $\mathbf{T}_{\mathbf{6}}\left(\mathbf{N}_{\mathbf{6 0}} \mathbf{P}_{\mathbf{3 0}} \mathbf{K}_{\mathbf{9 0}}\right)$ & 14.62 & 15.14 & 14.88 & 15.64 & 14.96 & 15.30 & 3.18 & 3.40 & 3.29 \\
\hline $\mathbf{T}_{\mathbf{7}}\left(\mathbf{N}_{\mathbf{6 0}} \mathbf{P}_{\mathbf{3 0}} \mathbf{K}_{\mathbf{1 2 0}}\right)$ & 15.54 & 15.81 & 15.68 & 15.75 & 15.20 & 15.47 & 3.21 & 3.37 & 3.29 \\
\hline $\mathbf{S ~ E m} \mathbf{\pm})$ & 0.44 & 0.47 & 0.33 & 0.37 & 0.61 & 0.36 & 0.16 & 0.16 & 0.11 \\
\hline $\mathbf{C D}$ at 5\% & 1.36 & 1.44 & 0.95 & 1.15 & 1.88 & 1.05 & 0.48 & 0.48 & 0.33 \\
\hline
\end{tabular}

Table.3 Effect of potassium fertilizer application on water soluble potassium (in ppm) in soils of kharif rice

\begin{tabular}{|l|l|l|l|l|l|l|l|l|l|l|}
\hline & \multicolumn{3}{|c}{ 45 DAT } & \multicolumn{3}{c|}{ 70 DAT } & \multicolumn{3}{c|}{ 90 DAT } \\
\hline Treatments & $2016-17$ & $2017-18$ & Pooled & $2016-17$ & $2017-18$ & Pooled & $2016-17$ & $2017-18$ & Pooled \\
\hline $\mathbf{T}_{\mathbf{1}}\left(\mathbf{N}_{\mathbf{6 0}} \mathbf{P}_{\mathbf{3 0}} \mathbf{K}_{\mathbf{0}}\right)$ & 10.23 & 9.85 & 10.04 & 9.70 & 9.57 & 9.64 & 9.21 & 8.52 & 8.86 \\
\hline $\mathbf{T}_{\mathbf{2}}\left(\mathbf{N}_{\mathbf{6 0}} \mathbf{P}_{\mathbf{3 0}} \mathbf{K}_{\mathbf{1 5}}\right)$ & 11.60 & 11.16 & 11.38 & 12.55 & 11.52 & 12.04 & 11.70 & 11.39 & 11.54 \\
\hline $\mathbf{T}_{\mathbf{3}}\left(\mathbf{N}_{\mathbf{6 0}} \mathbf{P}_{\mathbf{3 0}} \mathbf{K}_{\mathbf{3 0}}\right)$ & 15.90 & 15.22 & 15.56 & 15.91 & 14.95 & 15.43 & 15.36 & 14.24 & 14.80 \\
\hline $\mathbf{T}_{\mathbf{4}}\left(\mathbf{N}_{\mathbf{6 0}} \mathbf{P}_{\mathbf{3 0}} \mathbf{K}_{\mathbf{4 5}}\right)$ & 14.94 & 14.48 & 14.71 & 14.61 & 13.65 & 14.13 & 15.25 & 14.64 & 14.94 \\
\hline $\mathbf{T}_{\mathbf{5}}\left(\mathbf{N}_{\mathbf{6 0}} \mathbf{P}_{\mathbf{3 0}} \mathbf{K}_{\mathbf{6 0}}\right)$ & 17.98 & 17.78 & 17.88 & 17.69 & 16.83 & 17.26 & 16.96 & 16.32 & 16.64 \\
\hline $\mathbf{T}_{\mathbf{6}}\left(\mathbf{N}_{\mathbf{6 0}} \mathbf{P}_{\mathbf{3 0}} \mathbf{K}_{\mathbf{9 0}}\right)$ & 20.12 & 19.80 & 19.96 & 20.01 & 18.96 & 19.48 & 18.63 & 18.68 & 18.65 \\
\hline $\mathbf{T}_{\mathbf{7}}\left(\mathbf{N}_{\mathbf{6 0}} \mathbf{P}_{\mathbf{3 0}} \mathbf{K}_{\mathbf{1 2 0}}\right)$ & 23.33 & 23.07 & 23.20 & 23.25 & 22.68 & 22.97 & 22.57 & 22.15 & 22.36 \\
\hline $\mathbf{S ~ E m} \mathbf{\pm})$ & 0.53 & 0.84 & 0.51 & 1.08 & 0.92 & 0.71 & 1.14 & 1.07 & 0.78 \\
\hline $\mathbf{C D} \mathbf{a} \mathbf{5} \%$ & 1.64 & 2.58 & 1.49 & 3.34 & 2.84 & 2.08 & 3.51 & 3.28 & 2.28 \\
\hline
\end{tabular}


Table.4 Effect of potassium fertilizer application on exchangeable potassium (in ppm) in soils of kharif rice

\begin{tabular}{|l|l|l|l|l|l|l|l|l|l|}
\hline & \multicolumn{3}{|c|}{ 45 DAT } & \multicolumn{3}{c|}{ 70 DAT } & \multicolumn{3}{c|}{ 90 DAT } \\
\hline Treatments & $2016-17$ & $2017-18$ & Pooled & $2016-17$ & $2017-18$ & Pooled & $2016-17$ & $2017-18$ & Pooled \\
\hline $\mathbf{T}_{\mathbf{1}}\left(\mathbf{N}_{\mathbf{6 0}} \mathbf{P}_{\mathbf{3 0}} \mathbf{K}_{\mathbf{0}}\right)$ & 60.85 & 61.17 & 61.01 & 59.39 & 53.98 & 56.68 & 57.12 & 55.83 & 56.47 \\
\hline $\mathbf{T}_{\mathbf{2}}\left(\mathbf{N}_{\mathbf{6 0}} \mathbf{P}_{\mathbf{3 0}} \mathbf{K}_{\mathbf{1 5}}\right)$ & 68.51 & 69.62 & 69.07 & 67.17 & 65.69 & 66.43 & 65.56 & 65.72 & 65.64 \\
\hline $\mathbf{T}_{\mathbf{3}}\left(\mathbf{N}_{\mathbf{6 0}} \mathbf{P}_{\mathbf{3 0}} \mathbf{K}_{\mathbf{3 0}}\right)$ & 74.68 & 75.23 & 74.96 & 73.61 & 71.15 & 72.38 & 71.28 & 70.10 & 70.69 \\
\hline $\mathbf{T}_{\mathbf{4}}\left(\mathbf{N}_{\mathbf{6 0}} \mathbf{P}_{\mathbf{3 0}} \mathbf{K}_{\mathbf{4 5}}\right)$ & 75.19 & 76.33 & 75.76 & 74.09 & 75.03 & 74.56 & 71.38 & 67.03 & 69.20 \\
\hline $\mathbf{T}_{\mathbf{5}}\left(\mathbf{N}_{\mathbf{6 0}} \mathbf{P}_{\mathbf{3 0}} \mathbf{K}_{\mathbf{6 0}}\right)$ & 77.34 & 79.31 & 78.33 & 76.10 & 78.82 & 77.46 & 74.38 & 74.09 & 74.23 \\
\hline $\mathbf{T}_{\mathbf{6}}\left(\mathbf{N}_{\mathbf{6 0}} \mathbf{P}_{\mathbf{3 0}} \mathbf{K}_{\mathbf{9 0}}\right)$ & 78.45 & 78.03 & 78.24 & 76.82 & 79.11 & 77.97 & 75.12 & 71.30 & 73.21 \\
\hline $\mathbf{T}_{\mathbf{7}}\left(\mathbf{N}_{\mathbf{6 0}} \mathbf{P}_{\mathbf{3 0}} \mathbf{K}_{\mathbf{1 2 0}}\right)$ & 80.23 & 79.68 & 79.95 & 79.62 & 82.30 & 80.96 & 77.58 & 75.84 & 76.71 \\
\hline $\mathbf{S ~ E m} \mathbf{\pm})$ & 1.80 & 1.52 & 1.21 & 1.82 & 2.20 & 1.45 & 2.19 & 2.23 & 1.71 \\
\hline $\mathbf{C D} \mathbf{a t} \mathbf{5 \%}$ & 5.55 & 4.68 & 3.52 & 5.61 & 6.78 & 4.24 & 6.74 & 6.88 & 4.99 \\
\hline
\end{tabular}

Table.5 Effect of potassium fertilizer application on available potassium (in ppm) in soils of kharif rice

\begin{tabular}{|l|l|l|l|l|l|l|l|l|l|}
\hline & \multicolumn{3}{|c|}{ 45 DAT } & \multicolumn{3}{c|}{ 70 DAT } & \multicolumn{3}{c|}{ 90 DAT } \\
\hline Treatments & $2016-17$ & $2017-18$ & Pooled & $2016-17$ & $2017-18$ & Pooled & $2016-17$ & $2017-18$ & Pooled \\
\hline $\mathbf{T}_{\mathbf{1}}\left(\mathbf{N}_{\mathbf{6 0}} \mathbf{P}_{\mathbf{3 0}} \mathbf{K}_{\mathbf{0}}\right)$ & 71.07 & 71.03 & 71.05 & 69.09 & 63.55 & 66.32 & 66.33 & 64.35 & 65.34 \\
\hline $\mathbf{T}_{\mathbf{2}}\left(\mathbf{N}_{\mathbf{6 0}} \mathbf{P}_{\mathbf{3 0}} \mathbf{K}_{\mathbf{1 5}}\right)$ & 80.11 & 80.78 & 80.45 & 79.73 & 77.20 & 78.47 & 77.25 & 77.11 & 77.18 \\
\hline $\mathbf{T}_{\mathbf{3}}\left(\mathbf{N}_{\mathbf{6 0}} \mathbf{P}_{\mathbf{3 0}} \mathbf{K}_{\mathbf{3 0}}\right)$ & 90.58 & 90.45 & 90.51 & 89.52 & 86.11 & 87.82 & 86.64 & 84.33 & 85.49 \\
\hline $\mathbf{T}_{\mathbf{4}}\left(\mathbf{N}_{\mathbf{6 0}} \mathbf{P}_{\mathbf{3 0}} \mathbf{K}_{\mathbf{4 5}}\right)$ & 90.13 & 90.81 & 90.47 & 88.71 & 88.68 & 88.69 & 86.63 & 81.67 & 84.15 \\
\hline $\mathbf{T}_{\mathbf{5}}\left(\mathbf{N}_{\mathbf{6 0}} \mathbf{P}_{\mathbf{3 0}} \mathbf{K}_{\mathbf{6 0}}\right)$ & 95.32 & 97.09 & 96.20 & 93.78 & 95.64 & 94.71 & 91.35 & 90.40 & 90.87 \\
\hline $\mathbf{T}_{\mathbf{6}}\left(\mathbf{N}_{\mathbf{6 0}} \mathbf{P}_{\mathbf{3 0}} \mathbf{K}_{\mathbf{9 0}}\right)$ & 98.57 & 97.82 & 98.20 & 96.83 & 98.07 & 97.45 & 93.75 & 89.98 & 91.86 \\
\hline $\mathbf{T}_{\mathbf{7}}\left(\mathbf{N}_{\mathbf{6 0}} \mathbf{P}_{\mathbf{3 0}} \mathbf{K}_{\mathbf{1 2 0}}\right)$ & 103.56 & 102.75 & 103.16 & 102.87 & 104.98 & 103.93 & 100.15 & 97.99 & 99.07 \\
\hline $\mathbf{S ~ E m} \mathbf{\pm})$ & 1.69 & 2.09 & 1.36 & 1.96 & 3.00 & 1.81 & 2.61 & 2.52 & 1.92 \\
\hline CD at 5\% & 5.21 & 6.45 & 3.98 & 6.04 & 9.24 & 5.29 & 8.05 & 7.77 & 5.62 \\
\hline
\end{tabular}

Table.6 Effect of potassium fertilizer application on $\mathrm{HNO}_{3}$ soluble Potassium (in ppm) in soils of kharif rice

\begin{tabular}{|l|r|r|r|r|r|r|r|r|r|}
\hline & \multicolumn{3}{|c}{ 45 DAT } & \multicolumn{3}{c|}{ 70 DAT } & \multicolumn{3}{c|}{ 90 DAT } \\
\hline Treatments & $2016-17$ & $2017-18$ & \multicolumn{1}{|c|}{ Pooled } & $2016-17$ & $2017-18$ & \multicolumn{1}{|c|}{ Pooled } & $2016-17$ & $2017-18$ & Pooled \\
\hline $\mathbf{T}_{\mathbf{1}}\left(\mathbf{N}_{\mathbf{6 0}} \mathbf{P}_{\mathbf{3 0}} \mathbf{K}_{\mathbf{0}}\right)$ & 476.18 & 486.00 & 481.09 & 472.01 & 457.88 & 464.94 & 452.81 & 436.46 & 444.64 \\
\hline $\mathbf{T}_{\mathbf{2}}\left(\mathbf{N}_{\mathbf{6 0}} \mathbf{P}_{\mathbf{3 0}} \mathbf{K}_{\mathbf{1 5}}\right)$ & 500.04 & 495.04 & 497.54 & 516.95 & 525.88 & 521.42 & 530.52 & 541.47 & 536.00 \\
\hline $\mathbf{T}_{\mathbf{3}}\left(\mathbf{N}_{\mathbf{6 0}} \mathbf{P}_{\mathbf{3 0}} \mathbf{K}_{\mathbf{3 0}}\right)$ & 516.57 & 499.99 & 508.28 & 528.66 & 518.31 & 523.49 & 542.75 & 546.78 & 544.76 \\
\hline $\mathbf{T}_{\mathbf{4}}\left(\mathbf{N}_{\mathbf{6 0}} \mathbf{P}_{\mathbf{3 0}} \mathbf{K}_{\mathbf{4 5}}\right)$ & 524.95 & 506.51 & 515.73 & 550.48 & 533.30 & 541.89 & 566.28 & 563.90 & 565.09 \\
\hline $\mathbf{T}_{\mathbf{5}}\left(\mathbf{N}_{\mathbf{6 0}} \mathbf{P}_{\mathbf{3 0}} \mathbf{K}_{\mathbf{6 0}}\right)$ & 561.09 & 559.06 & 560.08 & 571.13 & 559.61 & 565.37 & 590.58 & 578.04 & 584.31 \\
\hline $\mathbf{T}_{\mathbf{6}}\left(\mathbf{N}_{\mathbf{6 0}} \mathbf{P}_{\mathbf{3 0}} \mathbf{K}_{\mathbf{9 0}}\right)$ & 571.64 & 558.08 & 564.86 & 579.22 & 592.01 & 585.62 & 594.01 & 622.52 & 608.27 \\
\hline $\mathbf{T}_{\mathbf{7}}\left(\mathbf{N}_{\mathbf{6 0}} \mathbf{P}_{\mathbf{3 0}} \mathbf{K}_{\mathbf{1 2 0}}\right)$ & 563.65 & 569.72 & 566.69 & 586.03 & 587.33 & 586.68 & 603.30 & 626.18 & 614.74 \\
\hline $\mathbf{S ~ E m} \mathbf{(} \mathbf{)}$ & 18.04 & 21.17 & 13.96 & 22.33 & 17.32 & 14.23 & 21.17 & 16.66 & 13.62 \\
\hline $\mathbf{C D}$ at 5\% & 55.60 & 65.23 & 40.75 & 68.80 & 53.37 & 41.54 & 65.22 & 51.33 & 39.75 \\
\hline
\end{tabular}


Similar results were recorded from experiments by Ranganathan and Sathyanarayana (1980), Venkatesh and Sathyanaryana (1994), Hirekurbaret al., (2000) and Padole and Mahajan (2003).

\section{Available potassium}

In study of the pooled data of 2016-17 and 2017-18 (table no 5) pertaining to available potassium, it was noticed that available potassium was significantly influenced by application of potassium fertilizer in all the treatments over the control. At 45 DAT highest available potassium was recorded in treatment $\mathrm{T}_{7}$ with a value of $103.16 \mathrm{ppm}$ which was significantly higher than rest of the treatments except treatment $T_{6}$ with which it was at par. At 70 and 90 DAT similar result were obtained from treatment $T_{7}$, which was significantly higher than all other treatments. The available potassium in control plot showed decreasing trend with time which was due to the depletion of potassium pool with plant uptake and leaching. Similar trend was observed from the pooled data of all the treatments except treatment $T_{7}$ where no such trend was discernible. Similar findings were obtained from Singh and Brar (1977) and Padole and Mahajan (2003)

\section{$\mathrm{HNO}_{3}$ soluble potassium}

$\mathrm{HNO}_{3}$ soluble Potassium from soil samples during kharif season of each year at 45, 70, and 90days after transplanting (DAT) were analyzed statistically and presented in the table 6. The pooled data suggests that $\mathrm{HNO}_{3}$ soluble Potassium was highly influenced by the application of potassic fertilizer in every stage i.e. 45, 70, and 90days after transplanting (DAT). At 45 DAT, treatment $\mathrm{T}_{7}$ found to be highest in terms of $\mathrm{HNO}_{3}$ soluble potassium and was at par with treatments $T_{5}$ and $T_{6}$ and significantly higher than all other treatments. Similar trend was also seen in soil samples of 70 DAT and 90 DAT. The range of $\mathrm{HNO}_{3}$ soluble potassium was within 481.09 to $566.69 \mathrm{ppm}, 464.94$ to $586.68 \mathrm{ppm}$ and 444.64 to $614.74 \mathrm{ppm}$ for 45 DAT, 70 DAT and 90 DAT respectively. Chand and Swami (2000) and Kaskar et al., (2001) recorded similar values from their experiments. $\mathrm{HNO}_{3}$ soluble Potassium found to be increasing in soil with fertilization over time whereas in treatment $\mathrm{T}_{1}, \mathrm{HNO}_{3}$ soluble potassium decreased with passing of time.

\section{References}

Bahmariar, M.A., Ranjbar, G.A. and Ahamafian, S.H., 2007. Effect of $\mathrm{N}$ and $\mathrm{K}$ application on agronomic characteristics of two Iranian and landrace rice (Oryza sativa L.) cultivars. Pakistan Journal Biological Science, 10(6): 880-886.

Bansal. S.K., Srinivas Rao, Ch., Pasricha, N.S. and Imas Patricia, 2002. Potassium dynamics in major benchmark soil series of India under long term cropping. $17^{\text {th }}$ WCSS, 14-21 August, Thailand, 1(20): 276.

Biswas, J.C., Ahmed, M.T. and Islam, M.R., 2001. Lodging vs. non-lodging in BRRI dhan. Bangladesh Journal of Training Development, 14(1-2): 107-113.

Black, C.A., Evans, D.D., Ensminger, L.E., White, J.L. and Clark, F.E., 1965. Methods of soil analysis (eds). American Society of Agronomy. Madison, Wisc. Agronomy (ASA). 09.

Chand, S. and Swami, B.N., 2000. Different forms of potassium in some important soils association of Bharatpur district of Rajasthan. J. of potassium Res., 16: 5961.

Gomez, K.A. and Gomez, A.A., 1984. Statistical Procedures for Agricultural Research 2nd Edition. John Wiley \& Sons. P. 680.

Hirekurubar, B.M., Satyanarayana, T., 
Sarangamath, P.A. and Manjunathaiah, H.M., 2000. Forms of potassium and their distribution in soils under cotton based cropping system in Karnataka. $J$. Indian Soc. Soil Sci., 48(3): 604-608.

Jackson, M. L., 1973. Soil chemical analysis. Prentice Hall of India Pvt. Ltd New Delhi; 183-93.

Kaskar, D.R., Salvi, V.G., Mayekar, B.S. and Dabke, D.J., 2001. Forms of potassium their interrelationship and relationship with other soil properties of Inceptisols of west coast of Maharashtra. J. of potassium res., 17: 23-27.

Krishnappa, M., Gowda, K.N., Shankarnarayan, V., Maharudrappa, K. and Khan, M.M., 2006. Effect of graded levels and split application of potassium on its availability and yield of rice. Journal of Potassium Research, 6(4): 156-161.

Mathad, K.K.V., Bandi, A.G. and Umesh, A., 2002. Influence of tillage, drainage practices and potassium levels on yield and quality of rice under tanked conditions. Research on Crops, 3 (3): 488-49.

Meena, S.L., Singh, S. and Shivay, Y.S., 2003. Response of rice (Oryza sativa L.) to $\mathrm{N}$ and $\mathrm{K}$ application in sandy clay loam soils. Indian Journal of Agricultural Science, 73(1): 8-11.1.

Mukherjee, D. and Sen, A., 2005. Influence of rice husk and fertility levels on the nutrient content of rice (Oryza sativa).
Department of Agronomy, Institution of Agricultural Science, Varansi, India. Agricultural Science, Digest.,25(2): 151-152.

Padole, V. R. and Mahajan, S. B., 2003. Status and release behavior of potassium in some swell-shrink soils of Vidarbha, Maharashtra. J. Maharashtra agric. Univ., 28(1):3-7.

Pharande, A.L. and Sonar, K.R., 1996. Depth wise distribution of different forms of potassium in important vertisol soil series of Maharashtra. J. Indian Soc. Soil Sci., 12(2):127-134.

Ranganathan, A. and Sathyanarayana, T., 1980. Studies on potassium status of soils of Karnataka. J. Indian Soc. Soil Sci.; 28:148-153.

Singh, B. and Brar, S.P.S., 1977. Dynamics of native and applied potassium in maizewheat rotation. Potash Rev., 9:1-6.

Tabar, S.Y., 2012. Effect of Nitrogen and Phosphorus Fertilizer on Growth and Yield Rice (Oryza sativa L). International journal of Agronomy and Plant Production, 3(12): 579-584, 2012.

Thakur, R.B., Jha, A.K. and Sharma, R.P., 1993. Effect of levels and times of $\mathrm{K}$ application on wetland rice. Proc. Int. Symp. New Delhi, India. p.138.

Venkatesh, M. S. and Satyanarayana, T., 1994. Status and distribution of potassium in vertisols of North Karnataka. J. Indian Soc. Soil Sci., 42(2): 229-233.

\section{How to cite this article:}

Sai Parasar Das, Goutam Kumar Ghosh, Suchhanda Mondal, Pabitra Kumar Biswas and Manik Chandra Kundu. 2019. Plant Growth Parameters and Soil Potassium Pool as Influenced by Potassium Fertilization in Kharif Season Rice. Int.J.Curr.Microbiol.App.Sci. 8(03): 1547-1553. doi: https://doi.org/10.20546/ijcmas.2019.803.178 